\title{
Steering of bright discrete photovoltaic solitons in lithium niobate waveguide arrays
}

\author{
Milutin Stepić ${ }^{1}$, Vladimir Shandarov ${ }^{2}$, Aleksandra Maluckov ${ }^{3}$, Feng Chen $^{1}$, Daniel Runde ${ }^{1}$, \\ Christian Rüter ${ }^{1}$, and Detlef Kip ${ }^{1}$ \\ 1 Institute of Physics and Physical Technologies, Technical University of Clausthal, \\ 38678 Clausthal-Zellerfeld, Germany; \\ 2 State University of Control Systems \& Radioelectronics, 40 Lenin Ave., 634050 Tomsk, Russia; \\ 3 Faculty of Sciences and Mathematics, University of Niš, 18000 Niš, Serbia and Montenegro
}

\begin{abstract}
A theoretical model which can describe the dynamics of spatial bright discrete solitons in photovoltaic photorefractive media is suggested. Four different types of staggered solitons, symmetric odd and even, twisted and antisymmetric odd one are observed numerically and their behavior is investigated with respect to small transverse phase offsets. A first experimental observation of steering of such symmetric odd staggered solitons in lithium niobate waveguide arrays is presented.
\end{abstract}

Keywords: discrete photovoltaic solitons, staggered solitons, nonlinear waveguide array, lithium niobate

\section{INTRODUCTION}

Spatial solitons are localized structures which can be found in various systems due to the interplay between diffractive and nonlinear effects, and especially optical spatial solitons are believed to have a great potential in future applications [1]. Photorefractive solitons, which arise from light-induced charge redistribution and internal space-charge fields that cause refractive index variations via the Pockels effect, require small optical power for their generation (microwatt level) and may have formation times down to the milliseconds range [2]. Photovoltaic solitons, which belong to this class of nonlinear waves, have been suggested to exist in media with a significant photovoltaic effect ten years ago [3] and soon thereafter they were observed in bulk lithium niobate crystals [4]. On the other hand, so-called discrete solitons, which result from the balance between discrete diffraction [5] and nonlinearity, were predicted to exist in nonlinear waveguide arrays in 1988 [6]. This generic type of soliton (discrete solitons are not directly related to a certain material) was experimentally observed in AlGaAs waveguide arrays six years ago [7]. Some years earlier it has been already suggested that discrete self-focusing can be achieved in a nonlinear waveguide array with defocusing nonlinearity [8]. However, up to date there are no results on discrete photovoltaic solitons and our aim is to bridge the gap between these two kinds of solitons.

The paper is organized as follows. In Section 2 we suggest a model equation, which can describe the dynamics of various discrete bright photovoltaic solitons and give the explicit expressions for each of them. Simulations which show the steering of these localized modes are presented in Section 3. Section 4 is devoted to the experimental setup and results, while conclusions are given in Section 5.

\section{THEORETICAL RESULTS}

\subsection{Model}

The scalar wave equation for the slowly varying amplitude of the optical field in steady state two-dimensional systems with a nonzero photovoltaic current and with electrons as the sole charge carriers is given by $[3,4]$ :

Tenth International Conference on Nonlinear Optics of Liquid and Photorefractive Crystals, edited by Gertruda V. Klimusheva, Andrey Iljin, Sergey Al. Kostyukevych, Proc. of SPIE Vol. 6023 60230K, (2005) - 0277-786X/05/\$15 - doi: 10.1117/12.648204 


$$
i \frac{\partial A_{x}}{\partial z}+\frac{1}{2 k} \frac{\partial^{2} A_{x}}{\partial x^{2}}+\frac{k \Delta n_{r}\left(A_{x}\right)}{n_{r 0}} A_{x}=0 .
$$

Here $k$ is the wave number and $n_{r 0}$ is the unperturbed extraordinary refractive index. Mathematically, the nonlinear refractive index perturbation due to the Pockels effect can be written as:

$$
\Delta n_{r}=\alpha \frac{I}{I+I_{D}}
$$

where $I=\left|A_{x}\right|^{2}$ is intensity, $I_{D}=G / q$ is the dark irradiance ( $G$ is the dark generation rate, while $q$ is the photoionization cross section), and $\alpha=-n_{r 0}{ }^{3} r E_{P V} / 2$. Here $r=r_{33}$ is the effective electro-optic coefficient, while $E_{P V}$ is the photovoltaic field constant. Using the following set of substitutions, $s=x / x_{0}, \xi=z /\left(k x_{0}{ }^{2}\right)$, and $A_{x}=U I_{D}{ }^{0.5}$, where $x_{0}$ is the arbitrary width, the wave equation (1) can be transformed into a dimensionless evolution equation:

$$
i \frac{\partial U}{\partial \xi}+\frac{1}{2} \frac{\partial^{2} U}{\partial s^{2}}-\beta_{P V} \frac{|U|^{2} U}{1+|U|^{2}}=0
$$

with $\beta_{P V}=k^{2} x_{0}{ }^{2}|\alpha| / n_{r 0}$. After the usual discretization this equation reads:

$$
i \frac{\partial U_{n}}{\partial \xi}+\frac{1}{2 h^{2}}\left(U_{n-1}+U_{n+1}-2 U_{n}\right)-\beta_{P V} \frac{\left|U_{n}\right|^{2} U_{n}}{1+\left|U_{n}\right|^{2}}=0
$$

where $U_{n}$ is the wave function of the $n$-th nonlinear element $(n=1, \ldots, N)$. In the case of periodic boundary conditions $\left(U_{N+1}=U_{1}\right), h=(L-N w) /\left(N x_{0}\right)$ is the normalized distance between two elements and $w$ represents the width of a single waveguide. This equation does not belong to the group of integrable equations because it has only two conserved quantities, power $P$ and Hamiltonian $H$, while the number of unknowns in this system of ordinary differential equations is $N$. The Hamiltonian is given by:

$$
H=\sum_{n=1}^{N}\left\{\beta_{P V}\left[\left|U_{n}\right|^{2}-\ln \left(1+\left|U_{n}\right|^{2}\right)\right]+\frac{\left|U_{n-1}-U_{n}\right|^{2}}{2 h^{2}}\right\},
$$

while the soliton power is given by:

$$
P=\sum_{n=1}^{N}\left|U_{n}\right|^{2}
$$

Our model equation will be examined in detail on the example of a waveguide array in the photorefractive crystal lithium niobate exhibiting a strong photovoltaic effect. The corresponding crystal parameters are $r=32 \times 10^{-12}$ $\mathrm{m} / \mathrm{V}, n_{r 0}=2.20$ (for the green light from the argon ion laser with $\lambda=514.5 \mathrm{~nm}$ ) while the photovoltaic field is assumed to be $25 \mathrm{kV} / \mathrm{cm}$. The arbitrary scaling length $x_{0}$ is set to $8 \mu \mathrm{m}$.

\subsection{Soliton solutions}

From the model equation (4) it is possible to get the following four approximate analytical expressions for the different narrow localized solutions:

a) symmetric odd staggered (o) solution reads:

$$
\left|U_{0}\right|=\sqrt{\frac{v-h^{-2}}{\beta_{P V}+h^{-2}-v}}, \quad\left|U_{0 \pm m}\right|=\frac{\left|U_{0}\right|}{2 h^{2}\left(v-h^{-2}\right)^{m}},
$$


where the central element of the array is marked with $n=0$, while $m=1,2, \ldots$

b)

The antisymmetric odd staggered (a) solution has the form [9]:

$$
U_{0}=0, \quad\left|U_{ \pm 1}\right|=\sqrt{\frac{v-h^{-2}}{\beta_{P V}+h^{-2}-v}}, \quad\left|U_{ \pm 1 \pm m}\right|=\frac{\left|U_{1}\right|}{2 h^{2}\left(v-h^{-2}\right)^{m}},
$$

c)

an even staggered (e) solution is described by:

$$
\left|U_{ \pm 1}\right|=\sqrt{\frac{v-1.5 h^{-2}}{\beta_{P V}+1.5 h^{-2}-v}}, \quad\left|U_{ \pm 1 \pm m}\right|=\frac{\left|U_{ \pm 1}\right|}{2 h^{2}\left(\nu-h^{-2}\right)^{m}},
$$

d) while twisted staggered ( $t$ ) solutions can be mathematically described by [9]:

$$
\left|U_{ \pm 1}\right|=\sqrt{\frac{v-0.5 h^{-2}}{\beta_{P V}+0.5 h^{-2}-v}}, \quad\left|U_{ \pm 1 \pm m}\right|=\frac{\left|U_{ \pm 1}\right|}{2 h^{2}\left(v-h^{-2}\right)^{m}} .
$$

These staggered solutions are shown in Fig. 1 for the soliton power $P=6.55$.
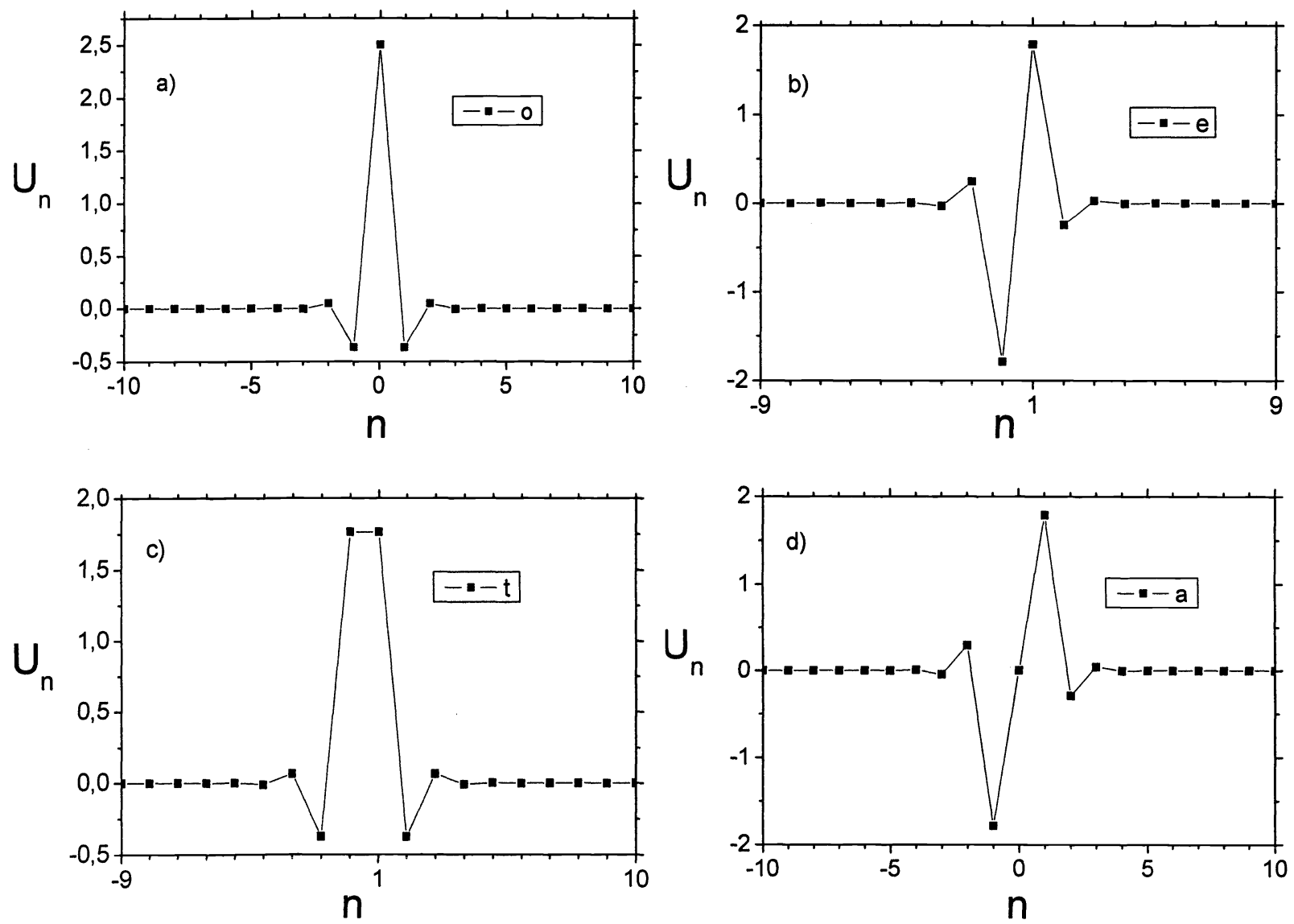

Fig. 1. Staggered discrete photovoltaic solitons with the same power $P=6.55$. a) Symmetric odd, b) even, c) twisted, and d) antisymmetric odd soliton. 


\begin{tabular}{|c|c|c|c|c|}
\hline$\left(0.5 h^{-2}, h^{-2}\right)$ & $\left(h^{-2}, 1.5 \mathrm{~h}^{-2}\right)$ & $\left(1.5 h^{-2}, \beta_{P V}+0.5 h^{-2}\right)$ & $\left(\beta_{P V}+0.5 h^{-2}, \beta_{P V}+h^{-2}\right)$ & $\left(\beta_{P V}+h^{-2}, \beta_{P V}+1.5 h^{-2}\right)$ \\
\hline $\mathrm{E}$ & $0, \mathrm{a}, \mathrm{t}$ & $0, \mathrm{a}, \mathrm{t}, \mathrm{e}$ & $0, \mathrm{a}, \mathrm{e}$ & $\mathrm{e}$ \\
\hline
\end{tabular}

Tab. 1. Frequency intervals for different types of narrow localized modes.

All of these solutions have a limited interval of allowed solitons frequencies. The width of these intervals depends on the values of $\beta_{S C}$ and $h^{-2}$. The corresponding frequency intervals where these analytical solutions can be found are given in Table 1.

In the linear regime, the last term in Eq. (4) can be neglected. An illustration of discrete diffraction of an even staggered solution in a waveguide array in lithium niobate is presented in Fig. 2.

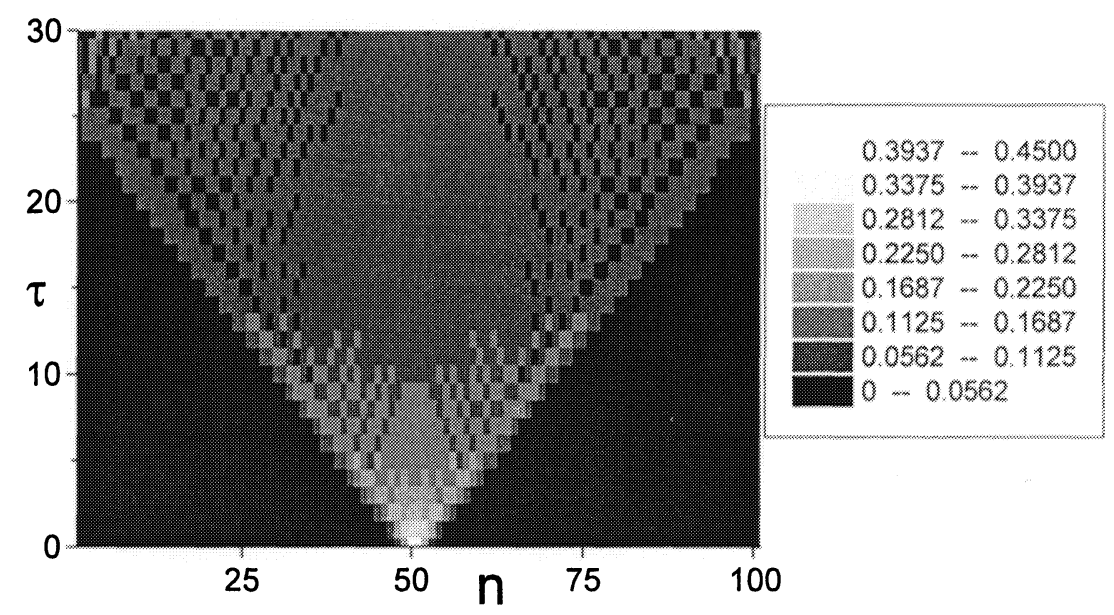

Fig. 2. Discrete diffraction of an even staggered mode in a one-dimensional lithium niobate waveguide array (top view). The number of elements in the array is $N=101$, while the initial value of the dimensionless pulse amplitude is 0.45 .

\section{NUMERICAL RESULTS}

In this section the linear stability of localized modes with respect to transverse translational shifts is considered. The localized modes are numerically forced to move transversally by introducing a small phase difference (phase tilt) $\varphi$ between adjacent lattice elements. The observed dynamics is rather complex and strongly depends on both soliton power and introduced phase offset. Some typical results are presented in Fig. 3. Twisted (Fig. 3e), odd symmetric (Fig. 3a) and odd antisymmetric solitons (Fig. 3g) may propagate stable along the array within the allowed interval of their power (frequency). An even soliton is usually trapped by the lattice and transformed into a symmetric odd breather [10] (Fig. 3b-c). Both twisted and odd antisymmetric solitons are unstable with respect to the introduction of a tiny phase tilt $\varphi$. Usually, these waves convert either into the corresponding symmetric odd breather with the same power and total energy, or evolve into a breather with lower energy where part of the energy is lost as radiation (Fig. 3f, h). Initially tilted symmetric odd solitons can be either trapped (Fig. 3b) or be unaffected by the lattice, i.e., a free propagation across the lattice may be obtained. 

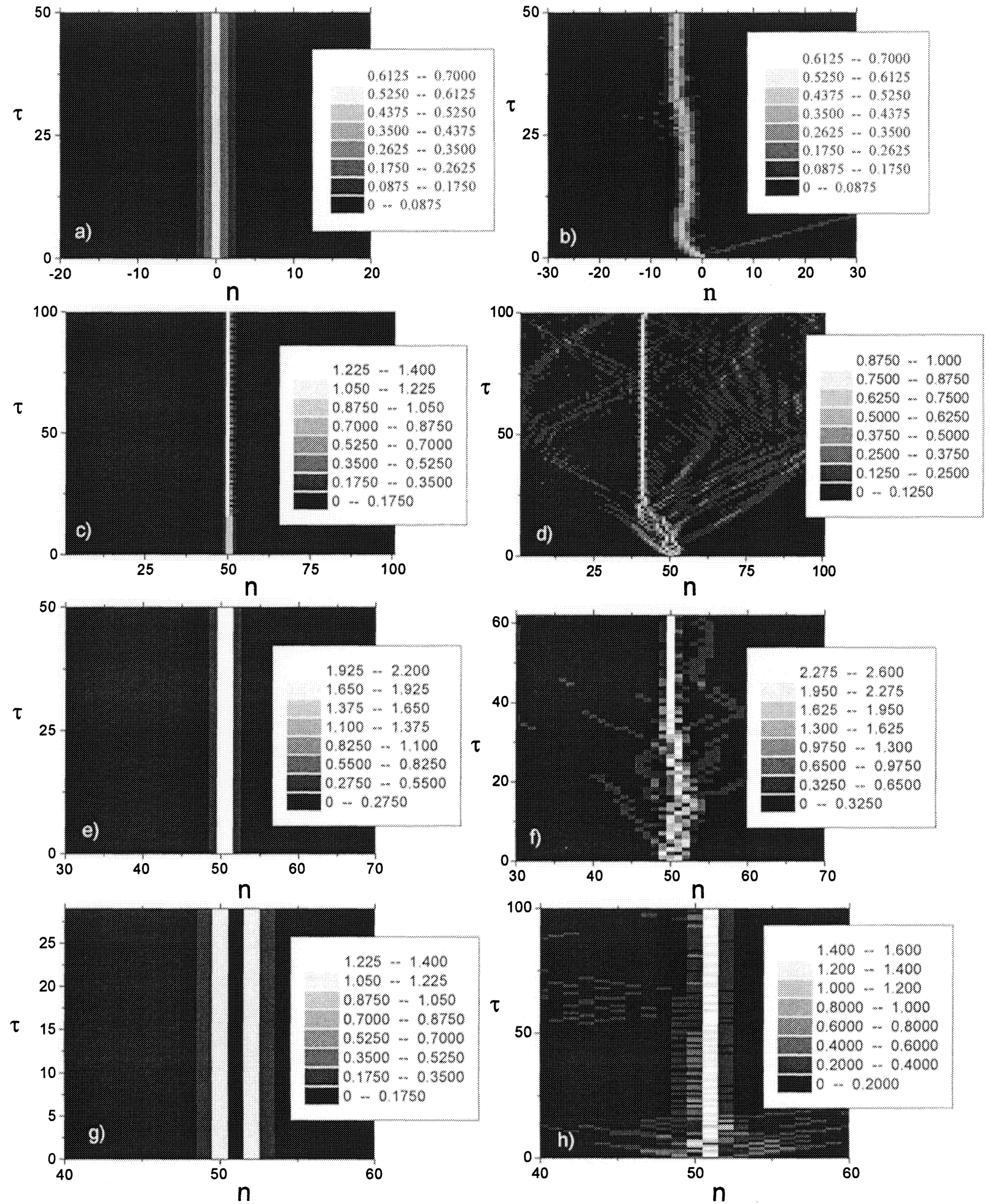

Fig. 3. Propagation of different localized modes with additional transverse phase offset. Symmetric odd soliton with $P=0.582$ a) $\varphi=0$, and b) $\varphi=0.25 \pi$. Even soliton with $v=15.28$ c) $\varphi=\pi$, and d) $\varphi=0.25 \pi$. Twisted staggered soliton with $v=17$ e) $\varphi=$ 0 , and f) $\varphi=0.25 \pi$. Antisymmetric odd soliton with $\nu=15.1 \mathrm{~g}) \varphi=0$, and h) $\varphi=0.5 \pi$. Here $\tau=\xi / 2 \mathrm{~h}^{2}$ is normalized propagation coordinate. 


\section{EXPERIMENTAL RESULTS}

In this section we describe our experimental setup that we have used and present results which confirms the steering effect of symmetric odd staggered solitons. Our experimental setup is sketched in Fig. 4. The green light of an argon ion laser $(\lambda=514.5 \mathrm{~nm})$ is divided into two beams by virtue of a Mach-Zehnder interferometer, where the optical power can be controlled by a combination of polarizer and half-wave plate. The two beams of equal power are overlapped under a small angle and are coupled into the waveguide array using a $40 \times$ microscope lens. The grating period of the interference pattern is carefully adjusted to match the inter-waveguide distance of the array. In this way a staggered input pattern, that consists of a central maximum and two first neighbours which are out of phase relative to the center, is obtained (see Fig. 5a). A cylindrical lens forms the elliptical shape of an input light beam to optimize the in-coupling efficiency. A CCD camera system serves for monitoring of the intensity distribution on the rear face of the array.

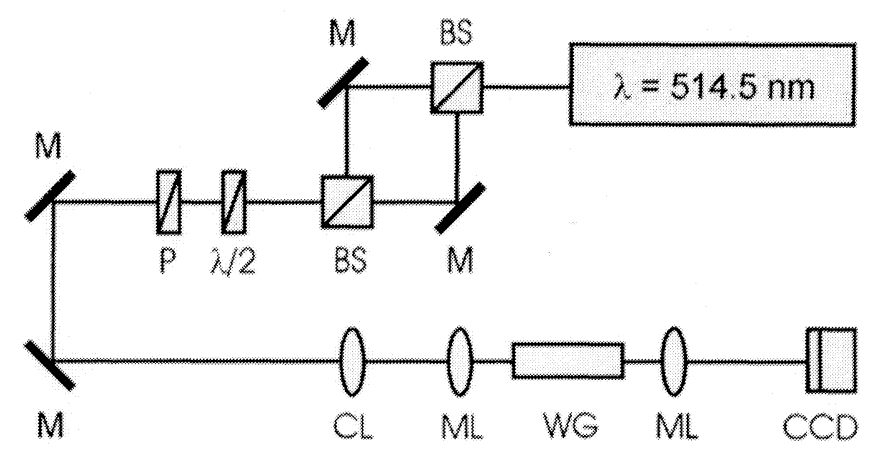

Fig. 4. Experimental setup. BS's, beam splitters; M's, mirrors; $\lambda / 2$, half wave plate; $\mathrm{P}$, polarizer; $C L$, cylindrical lens; ML's, microscope lenses; WG, waveguide array; CCD, CCD camera.

Our sample is fabricated by titanium in-diffusion [10] into $x$-cut substrates $(a \times b \times c=1 \mathrm{~mm} \times 7.8 \mathrm{~mm} \times 18 \mathrm{~mm})$ where the c-axis points along the grating vector. In order to enhance the photorefractive response, the sample was additionally doped by copper indiffusion with a copper concentration of $5 \times 10^{24} \mathrm{~m}^{-3}$. The distance between the adjacent channels is $3.6 \mu \mathrm{m}$ while the width of the single mode channel waveguides is $4 \mu \mathrm{m}$.

Both linear and nonlinear light propagation in the waveguide array is shown for an input power of about $4 \mu \mathrm{W}$ in Fig. 5. Because our sample has a rather large time constant, linear wave propagation can be observed immediately after switching on the input beams in Fig. 5B. This profile, which represents the regime of discrete diffraction, is slightly asymmetric due to a small initial phase offset of the two input beams. After a few minutes of illumination light-induced phase changes lead to self-focusing of the beam, which finally results in the formation of a steadystate odd symmetric staggered soliton (Fig. 5C), which is recorded up to steady state within one hour of illumination. This soliton is shifted with respect to the input beam demonstrating steering of the soliton across the array (compare with Fig. 3b). We were able to monitor this localized structure for almost two hours without any changes.

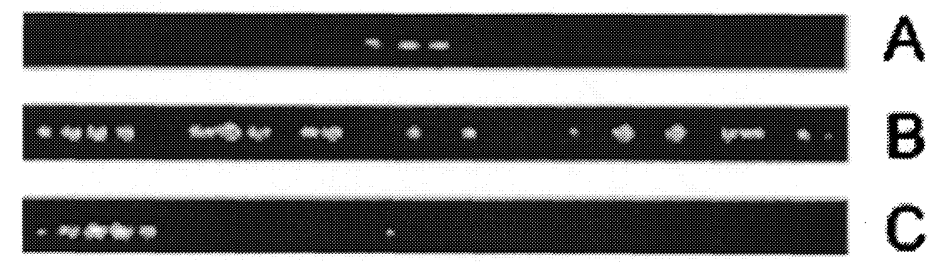

Fig. 5. Images from the $\mathrm{CCD}$ camera taken at the front $(\mathrm{A})$ and at the rear face $(\mathrm{B}, \mathrm{C})$ of the nonlinear waveguide array. 


\section{CONCLUSIONS}

In conclusion, the propagation of the bright spatial discrete solitons that result from the photovoltaic effect in a photorefractive material is modelled. It has been shown that self-focusing may be observed in an array of coupled defocusing nonlinear waveguides and that this leads to the creation of self-localized (soliton-like) patterns with relative phase difference of $\pi$ between neighbouring waveguides, so-called staggered localized modes. Four different solutions are found analytically: symmetric odd and even, twisted and antisymmetric odd staggered modes. Numerical calculations show the stable propagation of both symmetric and antisymmetric odd, and twisted staggered modes in the whole existence region, and the stable propagation of the low power even staggered mode. The steering properties of these localized modes are also considered and our results show that the even, the twisted, and the antisymmetric odd mode are unstable with respect to transverse phase offsets. The experimental observation of steering of stable odd symmetric soliton across the defocusing nonlinear waveguide array is also presented.

\section{ACKNOWLEDGMENTS}

This work is financially supported by INTAS (contract 01-0481), by the German Federal Ministry of Education and Research (BMBF, grant DIP-E6.1), by the Ministry of Science, Development and Technologies of Republic Serbia, Project 1964, and by the Alexander von Humboldt Foundation.

\section{REFERENCES}

1. V. E. Zakharov and S. Wabnitz, Optical solitons: Theoretical Challenges and Industrial Perspectives, Springer-Verlag, Berlin, 1999.

2. G. I. A. Stegeman, D. N. Christodoulides, and M. Segev, IEEE Journal on Selected Topics in Quantum Electronics 6, 1419 (2000).

3. G. C. Valley, M. Segev, B. Crosignani, A. Yariv, M. M. Fejer, and M. C. Bashaw, Phys. Rev A 50, R4457 (1994).

4. M. Taya, M. C. Bashaw, M. M. Fejer, M. Segev, and G. C. Valley, Phys. Rev A 52, 3095 (1995).

5. F. Lederer and Y. Silberberg, Opt. \& Photon. News 2, 48 (2002).

6. D. N. Christodoulides and R. I. Joseph, Opt. Lett. 13, 794 (1988).

7. H. S. Eisenberg, Y. Silberberg, R. Morandotti, A. Boyd, and J. S. Aitchinson, Phys. Rev. Lett 81, 3383 (1998).

8. Yu. S. Kivshar, Opt. Lett. 18, 1147 (1993).

9. F. Lederer, S. Darmanyan, and A. Kobyakov, Discrete Solitons. In Spatial Solitons, eds. S. Trillo and W. Torruellas, Springer Series on Optical Sciences, Vol. 82, Springer, Berlin, New York, 2001, pp. 269.

10. D. Kip, Appl. Phys. B 67, 131 (1998). 